\title{
Experimental study of the effects of Ulinastatin on reducing MDA in free flaps
}

\author{
Wei Lu*1, Ruixia ${ }^{*}{ }^{2}$, Yifan Sun ${ }^{1}$, Yafan Sun ${ }^{3}$, Mingjuan $\mathrm{Gu}^{1}$, Xiang Gao ${ }^{1}$, Peng $\mathrm{Yu}^{1}$, Jing Sun ${ }^{1}$ \\ ${ }^{1}$ Department of Plastic Surgery, Baogang Hospital, Baotou, Inner Mongolia, China \\ ${ }^{2}$ The Second Sanatorium for Retired Cadres of Shangdong Province, Jinan, China \\ ${ }^{3}$ Graduate Team of The Second Military Medical University, China
}

Received: June 25, 2016

Accepted: July 30, 2016

Online Published: September 10, 2016

DOI: $10.14725 /$ dcc.v3n3p9

URL: http://dx.doi.org/10.14725/dcc.v3n3p9

\begin{abstract}
Free flap transplantation is the most important plastic surgery treatment. Although free flap is an important tissue repair tool for plastic surgery, it is easy to happen due to the anastomotic stenosis or thrombosis, as well as the inflammatory reaction caused by ischemia-reperfusion injury of the flap and the inflammation caused by these inflammatory reactions. Microcirculation contracture or occlusion will lead to partial or complete flap necrosis, thus affecting the repair effect. Numerous studies show that neutrophils are closely involved in the process of ischemic injury of free flaps. The purpose of this experiment is to investigate the use of ulinastatin to inhibit the inflammatory infiltration of free flaps to improve the survival of the flap.
\end{abstract}

Key Words: Free flap, Ulinastatin

Free flap transplantation is the most important treatment method in the department of plastic surgery currently. Although free flap is used as an essential tool for tissue repair in the plastic surgery, it can happen easily that anastomotic stenosis or thrombosis, inflammatory reactions caused by flap ischemia-reperfusion injury and resulting microcirculation contracture even occlusion as transplantation of flaps requires vascular anastomosis. All the situations mentioned above can eventually lead to local or total necrosis of flaps, and then affect the repair effect. A large amount of researches have shown that neutrophils (NEUTs) are closely involved in the process of ischemia injury of free flaps. This experiment is intended to study the effect of Ulinastatin on improving flap survival (rate) by inhibiting inflammatory infiltration of free flaps. A marker enzyme in NEUT is chosen as an experimental indicator. The experiment is summarized as follows. China.

\footnotetext{
*Correspondence: Wei Lu; E-mail: dcc60@ncspress.com; Address: Department of Plastic Surgery, Baogang Hospital, Baotou, Inner Mongolia,
}

\section{Materials and methods}

\subsection{Materials}

Main drugs and reagents: Ulinastatin, $10^{5} \mathrm{U} /$ bottle (Guangdong Techpool Bio-pharma Co., Ltd.), MDA assay kits (Fuzhou Maixin Biotechnologies Development Company). Main experimental instruments: a multiple operating microscope (OLYMPUS, Japan), a variable-speed electric tissue tearor (Shanghai Non-standard Motors Co., Ltd.), a spectrometer (ZIMMER, USA). Experiment animals were 40 healthy adult New Zealand rabbits, weighed 2,000-2,500 g.

\subsection{Methods}

\subsubsection{Experimental animal grouping}

40 rabbits were randomly divided into two groups: the experiment group (U group) and the control group (D group). 
Each group consisted of 20 rabbits. Among them, 10 rabbits from each group were fed and then killed in $24 \mathrm{~h}$ after surgery for the detection of biochemical indexes. The other 10 rabbits from each group were fed for 10 days after surgery in order to observe flap survival. The rabbits were fed separately, free access to water, and fed by clean feed. The room temperature should be under control at around $25^{\circ} \mathrm{C}$.

\subsubsection{Flap design and operative technique}

Flap was designed as free flap model of latissimus dorsi on the back of rabbits (a rectangle of $5 \mathrm{~cm} \times 3 \mathrm{~cm}$ ). Before surgery, rabbits were required of back hair removal with an area of $5 \mathrm{~cm} \times 10 \mathrm{~cm}$, and received anesthesia by intraperitoneal injection with $2 \mathrm{ml} / \mathrm{kg}$ of Sumianxin ( 846 Mixture). After anesthesia, the rabbits were stabilized in prone position on the operating table. The operative site was sterilized with sterile drapes whisked, and then the skin and the hypodermis were incised along the designed line. Under the 5 $x$ operating microscope, the thoracodosal artery on the back of the rabbit was isolated by use of dissecting needle carefully and gently without the blood vessel exposed overly. Taking the exposed thoracodosal artery as the midpoint of one side, the flap was designed as a rectangle of $5 \mathrm{~cm} \times$ $3 \mathrm{~cm}$. After dissociation, the two ends of thoracodosal artery were clipped by use of the hemostat. After the blood vessel was cut off, it was anastomosed as it were with 11-0 nylon monofilament. Flap of latissimus dorsi was sutured in situ on the wound on the back of the rabbit. The drainage strip was placed under the flap with tie-over dressing employed partially. After surgery, the rabbits were given gentamicin injections $\left(8 \times 10^{4} \mathrm{U}\right)$ by IM to prevent from infection.

\subsubsection{Methods of administration}

The rabbits in $\mathrm{U}$ group were administered through the marginal ear vein by Ulinastatin (dissolved in normal saline) in 10 minutes before surgery. It was required that these rabbits were given injections on the time points of $3 \mathrm{~h}$ and $12 \mathrm{~h}$ after surgery. Single injected dose was $10^{5} \mathrm{U} / \mathrm{kg}$, the rabbits in $\mathrm{D}$ group were given equivalent dose of normal saline with the same method of administration.

\subsubsection{Measurement of malondialdehyde (MDA)}

10 rabbits were chosen from $\mathrm{U}$ group and $\mathrm{D}$ group respectively, and received anesthesia on the time point of $24 \mathrm{~h}$ after surgery in order to remove flap tissues from the distal place and the proximal place to the pedicles. Took these two places as midpoints and quickly cut off the flap from each place with an area of $1.0 \mathrm{~cm} \times 1.0 \mathrm{~cm}$. Flaps were rinsed out carefully with the surface moisture wiped. Under the condition of ice bath, flaps were made into 5\% tissue homogenate with the electric tissue tearor by $10 \mathrm{~s}$ per time ( 5 times in total). The tissue homogenate was quickly placed into the centrifuge for standard centrifugation $(3,000 \mathrm{r} / \mathrm{min}$, $15 \mathrm{~min}$ ). After centrifugation, the supernatant was taken and assayed by thiobarbituric acid (TBA) to measure the optical density at $532 \mathrm{~nm}$. The activity of MDA can be measured by calculation.

\subsection{Statistical methods}

Data results were represented by ( $\bar{x} \pm \mathrm{s})$, and the statistical software SPSS 10.0 was applied in the statistical analysis. Two-way analysis of variance for independent samples was applied to the comparison of the values of MDA between flap sections and between groups.

\section{Results}

\subsection{The measurement results of MDA in flap com- posite tissues in $\mathbf{2 4} \mathrm{h}$ after surgery}

The values of MDA in the proximal and distal section of the flap were compared within their respective groups. The difference $(p<.05)$ was of statistical significance. The level of MDA in the distal section was higher than that in the proximal section. As for the comparison between two groups, MDA concentration of each section in $U$ group was lower than that in $\mathrm{D}$ group. The difference $(p<.05)$ was of statistical significance. The results were shown in Table 1.

Table 1: Measurement results of MDA in flap composite tissues in $24 \mathrm{~h}$ after surgery $(\bar{x} \pm \mathrm{s})$

\begin{tabular}{lll}
\hline Group & Proximal Section & Distal Section \\
\hline U Group & $1.73 \pm 0.51$ & $3.83 \pm 0.77$ \\
D Group & $2.21 \pm 0.52$ & $5.15 \pm 0.98$ \\
\hline
\end{tabular}

\section{Discussion}

MDA, which is an indicator reflecting lipid peroxidation, is closely associated with free radical metabolism. Free radicals are essential destructive media in the process of ischemia injury. They are involved in the chain reaction and cause peroxidation in cells, the composition of internal membranes and proteins in the cells. Irreversible cell injuries can probably arise consequently. ${ }^{[1]}$ Lipid peroxidation is a process by which free radicals do damage to cells. The inter-reaction between lipids and free radicals lead to lipid peroxidation and the generation of related byproducts, resulting in the loss of membrane function and integrity. MDA is a type of aldehyde monomers degraded 
most from lipid peroxides after free radical attack. Unlike free radicals, MDA is stable and it is easier to measure its level by use of colorimetry. MDA has been used as a marker indicating the generation of free radicals, and its measurement has been applied to a large number of researches. Many researches have shown that NEUT is an important factor that aggravates the ischemia injury of flap. ${ }^{[2]}$ The infiltration of NEUT in the flap ischemia zone happens in the early stage of ischemia. In this study, the levels of MDA in the proximal section and the distal section were measured in $24 \mathrm{~h}$ after surgery. The measured activities of MDA had the same expression in $\mathrm{U}$ group and D group, and the levels of MDA appeared to be of significant difference, i.e., the level of MDA in the distal section was much higher than that in the proximal section. The results are identical with those got by other researchers. In the experiment, it was detected that the level of MDA in the distal section of flap was much higher than that in the proximal section of flap. Nevertheless, the distal section was the most serious part of flap ischemia-reperfusion injury, and it reflected that NEUT was significantly enriched in the most serious part of flap ischemia. Tissues in the distal place of flap were most seriously damaged. The phenomenon that NEUT was significantly enriched in the most serious part of tissue ischemia-reperfusion injury conforms to the theory of mechanism that NEUT is involved in the process of flap ischemia-reperfusion. ${ }^{[3]}$

As for the comparison between two groups, MDA concen-

\section{References}

[1] Gao C, Li R, Wang S. Ulinastatin protects pulmonary tissues from lipopolysaccharide-induced injury as an immunomodulator. J Trauma Acute Care Surg. 2012; 72(1): 169-176. PMid: 22002616.

[2] Sekine Y, Ko E. The influence of intraoperative oxygen inhalation tration of each section of flap tissues in U group was lower than that in D group, and the difference was of statistical significance. It is proven that the usage of Ulinastatin in the perioperative period can prevent NEUT from being enriched in the ischemic flap in the early stage. Combined with the phenomenon analysis that Ulinastatin appears to promote flap survival, it is indicated that the protective effect of Ulinastatin on the ischemic flap is realized by preventing NEUT from concentrating in the early stage and partially impeding NEUT in the flap from damaging tissues.

By analyzing the level of MDA in each section of flap in $24 \mathrm{~h}$ after surgery, the experiment is intended to study the effect of Ulinastatin (a protease inhibitor) by intravenous injection on free flap survival with the application of vascular anastomosis to rabbits. The results showed that: (1) The systemic usage of Ulinastatin in rabbits in the perioperative period can inhibit from inflammatory infiltration of NEUT in the free flap with the application of vascular anastomosis. (2) The mechanism of Ulinastatin to promote flap survival can probably be associated with the effect of impediment from NEUT, damaging ischemia-reperfusion tissues by preventing from NEUT infiltration and concentration in the ischemic tissues and inhibiting from the release of oxygen free radicals and other toxic substances.

\section{Conflicts of Interest Disclosure}

The authors have no conflicts of interest related to this article.

on patients with idiopathic pulmonary fibrosis. Masui. 2011; 60(3): 307-313. PMid: 21485100.

[3] Sato A, Kuwabara Y, Shinoda N, et al. Use of low dose dopamine, gabexate mesilate and ulinastatin reduces the water balance and pulmonary complication in thoracic esophagectomy patients. Dis Esophagus. 2005; 18(3): 151-154. PMid: 16045575. https:// doi.org/10.1111/j.1442-2050.2005.00485.x 